\title{
Invasiveness and Metastatic Aggressiveness in Small Differentiated Thyroid Cancers: Demography of Small Papillary Thyroid Carcinomas in the Swedish Population
}

\author{
Haytham Bayadsi $^{1} \cdot$ Martin Bergman $^{1} \cdot$ Malin Sund $^{1} \cdot$ Joakim Hennings $^{1}$
}

Published online: 13 December 2019

(C) The Author(s) 2019

\begin{abstract}
Background The western world is seeing a rising incidence of thyroid cancer. Improved diagnostic methods do not entirely explain this increase. Papillary thyroid carcinoma (PTC) is the most common subtype of thyroid cancer. Small PTC ( $\leq 20 \mathrm{~mm})$ and especially papillary thyroid microcarcinomas (PTMC $\leq 10 \mathrm{~mm}$ ) are considered to be lowrisk tumors but some cases are considerably more aggressive. Sufficient understanding of these mechanisms is a long-term goal for more efficient and safer treatment of these tumors.

Methods We identified 959 cases of small PTCs in the validated Scandinavian Quality Register for Thyroid, Parathyroid and Adrenal Surgery, grouped according to lymph node metastasis. These were analyzed according to age, gender, tumor size and geographic region.

Results Patients with N1b disease (lateral lymph nodes metastases) had a smaller tumor size compared to patients with N1a disease $(8.6 \mathrm{~mm}$ vs $10.1 \mathrm{~mm}$ respectively, $p<0.05$ ). Patients and specifically females with N1b disease were younger than those with N0 or N1a disease. Patients with N1b disease had a lower proportion of females $(60 \%)$ compared to N0 and N1a groups ( $81 \%$ and $78 \%$, respectively). The incidence of operated small PTCs and of lymph node engagement differs between geographic regions in Sweden.

Conclusions Small PTC and especially PTMC seem to show different patterns of aggressiveness and demography regarding lateral lymph node metastases and $7 \%$ had N1b disease and tumor $<1 \mathrm{~cm}$, underscoring the importance of lymph node evaluation in PTMC patients. More understanding of predictive factors, mechanisms for metastatic disease and causes of regional differences, is needed.
\end{abstract}

This paper was presented as an oral presentation at the IAES meeting/ 48th World Congress of Surgery August 11-15, 2019 in Kraków, Poland.

Electronic supplementary material The online version of this article (https://doi.org/10.1007/s00268-019-05312-4) contains supplementary material, which is available to authorized users.

Haytham Bayadsi

haytham.bayadsi@regionjh.se

1 Department of Surgical and Perioperative Sciences/Surgery, Umeå University, 90185 Umeå, Sweden

\section{Introduction}

Thyroid cancer (TC) is the most common cancer of the endocrine glands. Papillary thyroid carcinoma (PTC) constitutes the most common subtype of TC (85-90\%) and generally has a good prognosis with disease-free, 5-year survival (DFS) at $87.5 \%$ and cancer-specific mortality after 5 years at $0.8 \%$. In differentiated papillary thyroid carcinoma, the 10-year survival rate ranges between 85 and $95 \%$ [1-5]. The current treatment for PTC consists of total thyroidectomy complemented by postoperative radioactive iodine and thyroxine suppression. For papillary thyroid 
microcarcinomas (PTMC, $\leq 10 \mathrm{~mm}$ ), hemithyroidectomy alone is considered sufficient $[6,7]$.

In recent decades, a rapid increase in TC incidence has been reported worldwide and, in some countries, TC is the fastest growing form of all cancers, especially in women [5, 8-10]. Incidence is steadily increasing. However, mortality rate is relatively unchanged, and the prognosis is still good for differentiated thyroid carcinoma $[1,6,10]$. Small PTC ( $\leq 20 \mathrm{~mm}$ in size) and especially papillary thyroid microcarcinomas (PTMC $\leq 10 \mathrm{~mm}$ ) are responsible for the majority of the incidence rate increment $[5,6]$. One etiologically established risk factor is ionizing radiation, but this alone cannot explain the increasing incidence of PTC $[6,11]$. Another contributing factor is believed to be the widespread use of ultrasound examinations of the neck and fine-needle aspiration cytology (FNAC) of lesions found in the thyroid gland. FNAC leads to increased diagnosis, but also makes it possible to diagnose patients at an early stage [7, 10, 12]. Other contributing factors like genetic mutations (for example BRAF) and geographic differences in iodine supply can affect the incidence of various TC types [6].

Small PTCs are classified as low-risk or extremely lowrisk tumors with favorable prognosis. Yet, a few of these can be more aggressive, causing lateral lymph node metastases, distant metastases or even death, possibly requiring another treatment strategy $[6,7,12,13]$. These findings indicate that there is a difference in biological aggressiveness within this group of tumors. Known histological features that are associated with a worse prognosis are multifocality, bilateral foci, vascular invasion, extrathyroidal extension and/or lymph node metastases. Regional lymph node metastases are a predictor for recurrence and patients with lymph node metastases have an increased risk of death from differentiated papillary thyroid cancer, especially if the tumor size is $>4 \mathrm{~cm}$ $[12,14,15]$. Age has classically been considered a strong predictive factor in carcinoma death, but is now more often considered in conjunction with other variables $[6,16]$. Therefore, decisions regarding treatment can be very difficult for physician and patient alike, especially in lesions below $1 \mathrm{~cm}$ without aggressive features. In such cases, active surveillance can be used as a treatment method in order to avoid the side effects of possibly unnecessary surgery $[12,13,17]$.

Thus, there is a great clinical need to establish factors related to invasiveness in small PTCs and to characterize the demographic as well as histological and molecular differences between the low- and high-risk forms of PTC. The aim of this study is to analyze small PTC with and without lymph node metastases, which is a marker for aggressiveness, using the Scandinavian Quality Register for Thyroid, Parathyroid and Adrenal Surgery (SQRTPA)
[1], with the aim of discovering potential demographic differences between these groups.

\section{Materials and methods}

\section{Selection of the cohort from SQRTPA}

Our study is a registry-based retrospective observational cohort study based on a validated and prospectively maintained registry - the Scandinavian Quality Register for Thyroid, Parathyroid and Adrenal Surgery (SQRTPA) [1]. The register was established in 2004 and is the world's first quality registry for endocrine operations. The registry has a covering range of almost $100 \%$ for thyroid surgery and is validated against the national patient registry and is one of the few registries that have an internal quality audit that randomly controls the operating centers every year [1]. Almost all thyroid operations performed in Sweden are registered in SQRTPA. The register does not, however, provide longitudinal long-term follow-up data for followup regarding local and distant recurrence. The study was approved by the Umeå Regional Ethics Committee, permit number 2016/254-31. Data between January 2011 and December 2015 were included. The registry specifies in which hospital the surgery was performed. Patients are sometimes sent to hospitals other than the one nearest their home, but patients are very seldom sent between regions. Thus, an examination at the regional level gives an accurate depiction of PTC cases. We therefore chose to examine the regional level and correlated each hospital to its region according to the six Swedish medical regions (Northern, Uppsala-Örebro, Stockholm, South-Eastern, Western, Southern). Supplemental Table 1 shows the distribution of the regional population in 2015 [18].

The inclusion criteria were a primary diagnosis of small PTC, defined as a tumor of $\leq 20 \mathrm{~mm}$ in size and T1 stage. These selected $\mathrm{T} 1$ cases were subsequently divided into additional subgroups according to lymph node status staging, namely a group without lymph node metastases (N0 group) and another with lymph node metastases (N1 group). The N1 group was further divided into N1a and $\mathrm{N} 1 \mathrm{~b}$ subgroups based on the location of metastases, where $\mathrm{N} 1 \mathrm{a}$ is classified as metastasis to the central cervical lymph nodes (level VI) and N1b is classified as metastases to the ipsilateral, bilateral or contralateral cervical lymph nodes (level I-V). The inclusion was based on the tumor-nodemetastasis (TNM) classification (TNM 7th edition) [19]. Tumors $\leq 20 \mathrm{~mm}$ classified as T3 have been debated and now re-classified in TNM 8th edition, and thus most of these tumors would be down-staged to T1 tumors according to size. As we are not able to differentiate this in the register, we have chosen to exclude these cases $(n=83)$ in 
order to have a histopathologically validated $\mathrm{T} 1$ cohort. No T4 tumors $<20 \mathrm{~mm}$ were registered in the SQRTPA during the period. Nx classified tumors (no lymph node could be found) are clinically and prognostically treated as N0 groups and therefore were included in the NO group.

The prevalence of distant metastases (M1) was, as expected, very low in the cohort ( $n=3$ in the N0 as well as in the N1 group) and was considered too low to be further analyzed.

\section{Statistics}

The aim was to determine whether there were any significant demographic differences between the groups based on age, gender, tumor size and geographical location of patients.

When assessing the tumor sizes, the Kruskal-Wallis test was used to compare between different groups. The Chisquare test was used for comparison between different genders. The one-way ANOVA test was used for comparison between different age groups. The statistical analyses were performed using SPSS version 25. The graphs were created using Graphpad Prism, 8th version.

\section{Results}

\section{Description of the cohort}

Of the 14,827 thyroid surgeries that were performed during the study period, 1004 patients were identified according to the inclusion criteria. Ten patients were excluded due to data discrepancy as they had been given TNM classification $\mathrm{T} 1$ but the tumor size registered was outside of the 1-20 mm, as were all T1 classified tumors with no tumor size noted in the registry $(n=35)$. This left a total of 959 cases analyzed in the study, as shown in Fig. 1.

\section{Regional differences of surgery for small PTC in Sweden in relation to $\mathbf{N}$ status}

When analyzing the distribution of the number of patients who underwent surgery for small PTC per 100,000 inhabitants in each specific region, we could observe differences between regions within the N0 and N1a subgroups as well as in the proportion of patients operated on for N1b metastases compared with N0 and N1a subgroups (Fig. 2). The proportion of $\mathrm{N} 1$ disease operated patients was the highest in Stockholm region with 4 operations/100,000 inhabitants (even for both N1a disease 2.8 and N1b disease 1.22 operations/100,000 inhabitants). Stockholm region also had the lowest N0 to N1 disease ratio (2.18). The N1a disease-to-N1b disease ratio was the highest in Western and Southern region, in which the proportion of the N1b was quite low (Supplementary Table 1).

\section{Types and indications for the performed surgeries}

In the N0 disease group, 423/739 (57\%) patients underwent a primary operation due to confirmed or suspected malignancy (182 with total thyroidectomy, 233 with hemithyroidectomy and eight with isthmus or subtotal resection) and $316 / 739(43 \%)$ were operated due to presumed benign indication (198 with total thyroidectomy, 101 with hemithyroidectomy and 17 with isthmus or subtotal resection). In the N1 disease group, 216/220 (98\%) patients were operated due to confirmed or suspected malignancy (173 with total thyroidectomy, 39 with hemithyroidectomy and four patients with isthmus or subtotal resection). Four patients $(2 \%)$ in the $\mathrm{N} 1$ disease group were operated without suspicion of malignancy (two with total thyroidectomy and two with hemithyroidectomy).

Surgery was performed according to unchanged national guidelines during the study period including the recommendation for central lymph node dissection in cases with a preoperatively known TC (of any size) and/or clinically noted central lymph node metastases during surgery, and lateral node dissection if lymph node metastases were proven in the lateral compartment, i.e., overall a more aggressive approach than the more selective central node clearance advocated by the newer national guidelines after the latest American Thyroid Association (ATA) revision to lower the risk for postoperative hypoparathyroidism. However, the registry does not give reliable data concerning intention for the central compartment surgery (diagnostic/prophylactic/therapeutic); so we could not further sub-analyze this within the study.

\section{Differences between groups in relation to $\mathbf{N}$ status}

Patients with N1 disease had a statistically significant larger tumor size when compared to patients with N0 disease as a whole $(7.9 \mathrm{~mm}$ vs. $10.1 \mathrm{~mm}$ respectively, $p<0.05)$. When further looking at the N1 subgroups N1a and N1b, patients with N1a disease had a significant larger tumor size compared with the patients with N1b disease (10.7 mm vs. $8.6 \mathrm{~mm}$, respectively, $p<0.05)$. Further comparison showed no significant difference in the tumor size between the disease groups N0 and N1b, although both these groups retained a significant difference versus the N1a disease subgroup, as shown in Fig. 3 and Table 1.

The ages of patients follow the same pattern as seen in the Swedish National Guidelines for Thyroid Cancer, peaking at 41-50 years of age. However, patients and specifically females with N1b disease were younger and stands out with an earlier peak at 31-40 years of age with a 
Fig. 1 Patient selection

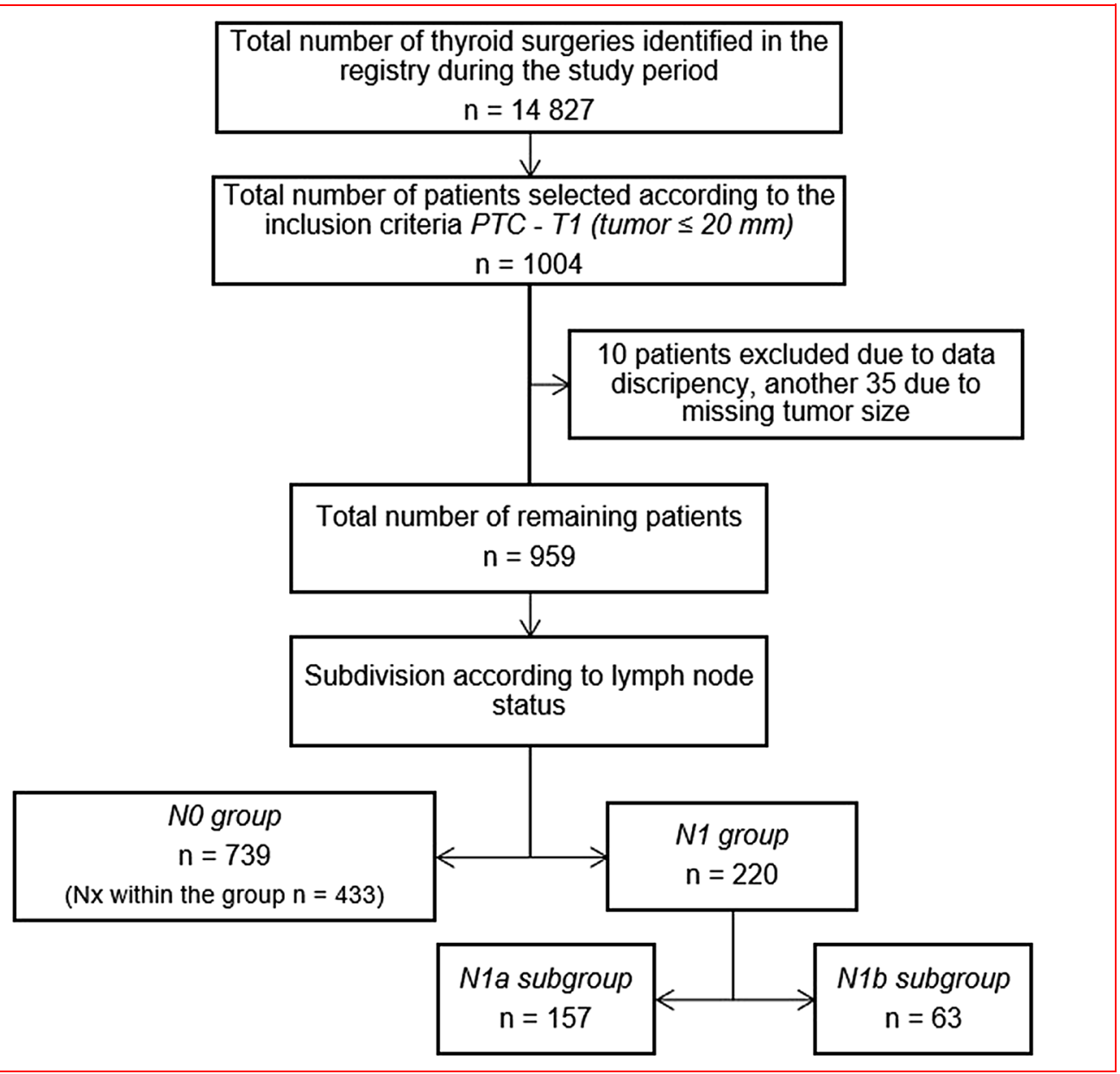

lower median (41 years) compared with the other groups, as shown in Fig. 4d and Table 1. The patients in the N0 disease group were more likely to be females (81\%) with a higher age mean and median (50.2 and 50 years, respectively, $p<0.01)$ compared to the other groups. When comparing the proportions of the two genders between the groups, the proportion of females in N1b disease subgroup was lower $(60 \%)$ compared with the $\mathrm{N} 0(81 \%)$ and N1a (78\%) groups, and stands out significantly as being disproportionate $(1: 1.52, p<0.05)$ in contrast to the $3: 1$ female/male ratio for TC described in the literature $[10,20]$, as given in Table 1 .

\section{Discussion}

In this observational study from a validated national quality registry, we found some regional differences in the incidence of small PTCs. We plan to investigate the reasons for this in a future study. Environmental factors might contribute as well as a combination of tumor-promoting elements. This retrospective, demographic register study cannot distinguish whether these incidence differences are due to environmental causes or whether, at least in part, they are influenced by differences in diagnostic work-up and the frequency of thyroid surgery performed in different regions of Sweden.

Stockholm region had the lowest N0 to N1 disease ratio (2.18), which might indicate a difference in the distribution of patients with N0 and N1 disease in Stockholm region compared with the other regions, which we speculate due to Stockholm region being the capital region with younger inhabitants compared with the other regions, and as showed before, the N1 disease had an earlier younger age peak.

The incidence difference between N0 and N1a status may also to a small part be influenced by some local variations in the application of the National Guidelines for Thyroid Cancer but we have to believe and do expect that centers follow the guidelines. These, however, were the same for the entire study period and included the recommendation for (a) central lymph node dissection in cases with a preoperatively known TC and/or clinically noted central metastases during surgery, and (b) lateral node dissection if metastases were proven in the lateral compartment. Less extensive surgical applications in various localities depending on, for example, old age or 


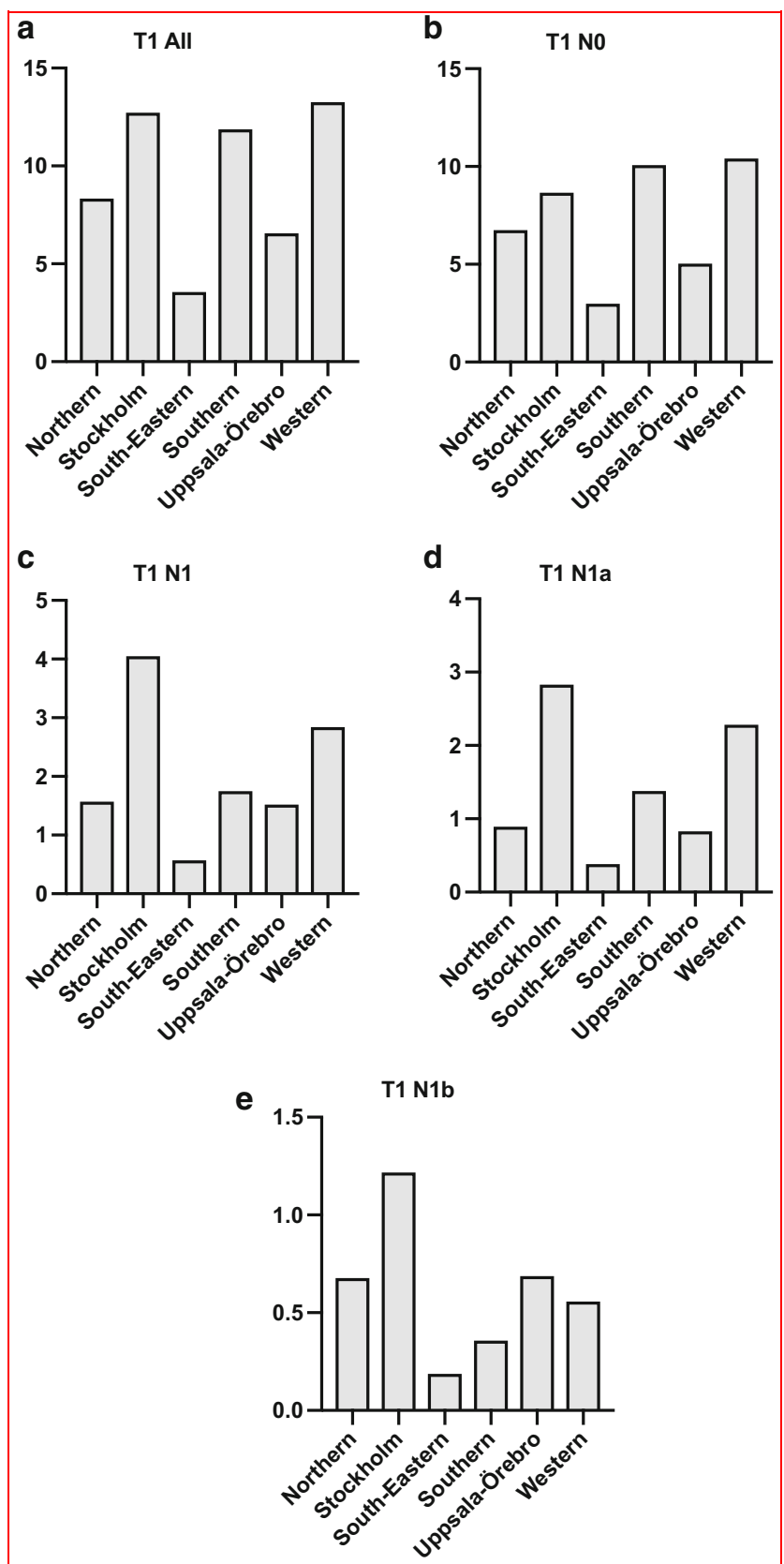

Fig. 2 Number of patients operated on per 100000 inhabitants for each region. a The distribution according to $\mathrm{T} 1$ without regard to the nodal status. b-e The distribution according to the different lymph node subgrouping

comorbidity and the surgeon's decision, can also contribute to some minor extent. According to the National Guidelines during the study period, there was no recommendation for re-interventive, central lymph node surgery within a two-stage surgical procedure if the TC was preoperatively unknown and the tumor was small.

However, the geographic differences in the N1b group are unlikely to be affected by these circumstances and are more likely to represent the aggressive tumors that are more amenable to metastasis unless adequate lymph node work-up, and appropriate node surgery is undertaken and well-adopted in all geographic regions alike.

Even though a formal sub-analysis of the causes of these regional discrepancies was not possible on SQRTPA register data and was not within the main scope of this demographic descriptive study, these findings, in our opinion, warrant further studies comparing geographic, environmental and tumor-promoting factors comparing the data with the Swedish Cancer Register as well as using modern geographic and environment analyzing software, and we plan to do this in a follow-up study.

We found an association between the tumor size and increasing lymph node metastases (N1 disease group) in small PTCs, indicating (as expected) that a larger tumor is generally more aggressive and thus increases the risk for lymph node metastases. This is in accordance with the literature [3, 6, 21]. However, when analyzing the N1a and N1b groups separately, tumor size was significantly smaller in the N1b group, indicating a possibly different mechanism for lateral lymph node engagement than that of central node metastases, the latter more predicted by tumor size. A comparison between patients with both central and lateral lymph node metastases vs patients with only lateral metastases would be interesting but, unfortunately, is impossible to obtain from the register. Further studies are warranted to determine whether this is related to a different tumor biology of certain small PTCs, such as mutations (e.g., BRAF) or different growth factors within the tumor stroma, multifocality and the topical location (polarity and laterality) of the tumor within the thyroid gland or a combination of all these factors that facilitate the development of lateral metastases in the setting of a small PTC $[12,22]$. We have initiated such studies on the cohort on both stromal markers and BRAF mutations, but data remain to be evaluated. BRAF mutations alone cannot be held responsible for tumor aggressiveness according to our preliminary data, though. One can also, of course, speculate if a selection bias may contribute in some cases as lateral lymph node metastases occasionally occur in the absence of a primary tumor in the thyroid though meticulous histopathological examination as well as an incidental PTMC might have been noted on histopathology not being related to the lateral lymph node metastasis.

Our finding that approximately $7 \%$ of patients with PTMC and thus tumor size $<10 \mathrm{~mm}$ without local invasion show lateral lymph node metastases at the time of primary surgery enlightens the need of a thorough preoperative lymph node evaluation and also stresses the importance of concomitant lymph node evaluation when applying a watchful waiting and in some cases avoidance of FNAC in small PTCs as new international as well as national guidelines advocate. 


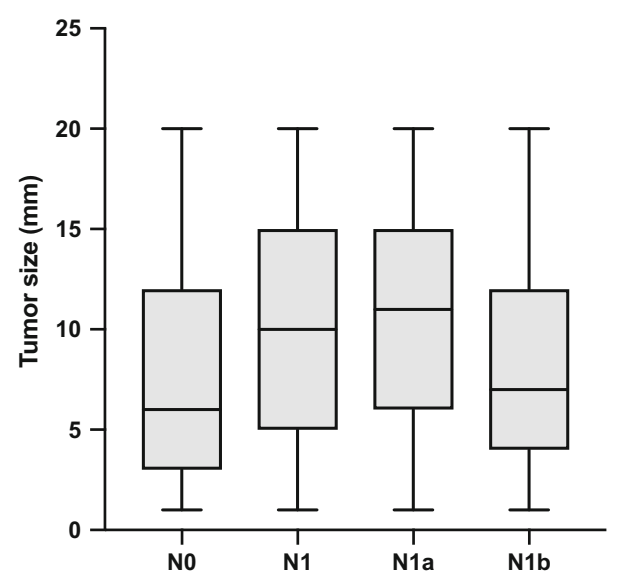

Fig. 3 Distribution of the tumor sizes in each lymph node subgroup according to the $\mathrm{N}$ classification ( $X$-axis, number of patients). The N1 disease group has a significantly larger tumor size $(10.1 \mathrm{~mm})$ on average when compared with the N0 disease group $(7.9 \mathrm{~mm})$ $(p<0.05)$. The N1b disease subgroup has a significantly smaller tumor size $(8.6 \mathrm{~mm})$ on average compared with the N1a disease subgroup $(10.7 \mathrm{~mm})(p<0.05)$. The Kruskal-Wallis test was used to compare the different groups. The whiskers represent the lower and upper tumor size limits (1 and $20 \mathrm{~mm}$, respectively)

Male gender is a known negative predictive factor in overall PTC prognosis [3] and was also observed to be correlated with $\mathrm{N} 1 \mathrm{~b}$ disease subgroup in our study in which the proportion of females was significantly lower. However, the N1b group is relatively small and a larger cohort would be of value to confirm these results. This is also needed to establish whether there is an effect in relation to recurrent disease and mortality.

In terms of age, we found a statistical difference between the N0 and $\mathrm{N} 1$ groups as a whole, and in the female subgroup specifically, when analyzing the subgroups separately. A probable reason for this would be that many cases in which the small PTC was found consist of patients who underwent surgery for nodular goiter with preoperatively unknown cancer; a disease clearly dominated by postmenopausal women. In these cases, no routine lymph node dissection was carried out in the absence of enlarged or suspicious lymph nodes, so most of this group fall into the N0 group. According to earlier Swedish data presented by Lundgren et al. [3], the incidence peak for thyroid cancer (all TC types included) among women was at 55-65 years. This was similar for men, whereas we noted a peak for almost all categories at $41-50$ years of age for T1 PTC. The time period in that study ranged from 1958 to 1987 . This difference in age peak could possibly be explained by the development of better diagnostic methods, which produce earlier diagnosis. It could also indicate that the incidence age has indeed moved to a younger demographic distribution.

\section{Conclusion}

Tumor size in general correlates with a more aggressive disease. However, there was a strong correlation between the N1b subgroup (representing the more aggressive

Table 1 Distribution of gender, age and tumor diameter (size) between the different lymph node subgroups according to the $\mathrm{N}$ classification

\begin{tabular}{|c|c|c|c|c|}
\hline & N0 group & N1 group & N1a group & N1b group \\
\hline Total number of patients & $739(77 \%)$ & $220(23 \%)$ & $157(16 \%)$ & $63(7 \%)$ \\
\hline Females & $597(81 \%)$ & $160(73 \%)$ & $122(78 \%)$ & $38(60 \%)$ \\
\hline Males & $142(19 \%)$ & $60(27 \%)$ & $35(22 \%)$ & $25(40 \%)$ \\
\hline Male/female ratio & $1: 4.2$ & $1: 2.67$ & $1: 3.49$ & $1: 1.52$ \\
\hline \multicolumn{5}{|l|}{ Age (years) } \\
\hline Mean & 50.2 & 45.6 & 45.8 & 45.2 \\
\hline Median & 50 & 44 & 45 & 41 \\
\hline Range & $14-88$ & $12-91$ & $12-91$ & $12-91$ \\
\hline \multicolumn{5}{|l|}{ Tumor diameter (mm) } \\
\hline Mean & 7.9 & 10.1 & 10.7 & 8.6 \\
\hline Median & 6 & 10 & 11 & 7 \\
\hline Range & $1-20$ & $1-20$ & $1-20$ & $1-20$ \\
\hline
\end{tabular}

Male-female ratio analysis shows that the female proportion in the N1b disease group is lower compared with the other groups $(p<0.01)$. The Chi-square test was used for gender comparisons. The N1 disease group has a significantly larger tumor size (10.1 mm) on average when compared with the N0 disease group $(7.9 \mathrm{~mm})(p<0.05)$. The N1b disease subgroup has a significantly smaller tumor size $(8.6 \mathrm{~mm})$ on average compared with the N1a disease subgroup $(10.7 \mathrm{~mm})(p<0.05)$ (as shown in Fig. 3) 
Fig. 4 Age distribution according to different lymph node subgroups according to the $\mathrm{N}$ classification. The age intervals are indicated on the $X$ axis. The number of patients is indicated on the $Y$-axis. d shows that the patients with $\mathrm{N} 1 \mathrm{~b}$ disease subgroup were younger and stands out with an earlier peak at $31-40$ years (black bar)

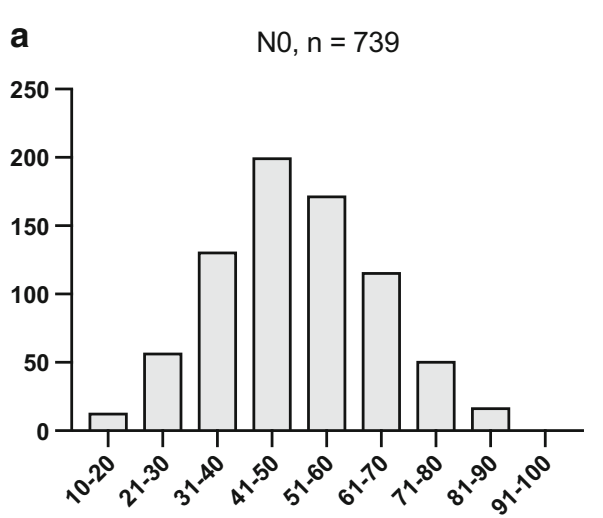

b $\quad \mathrm{N} 1, \mathrm{n}=220$

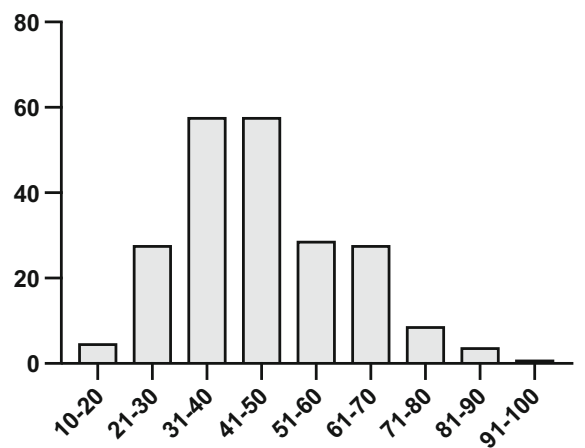

C

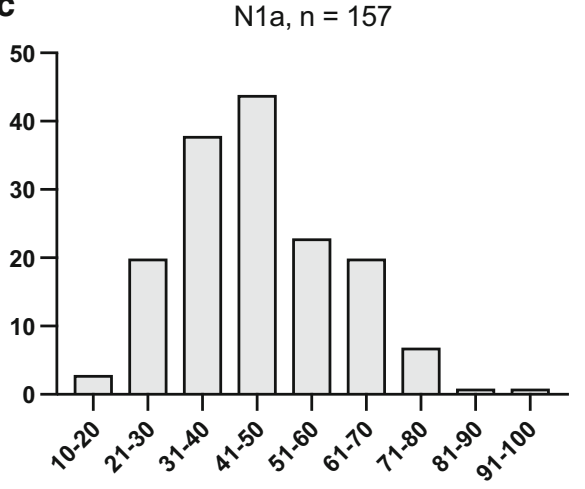

d

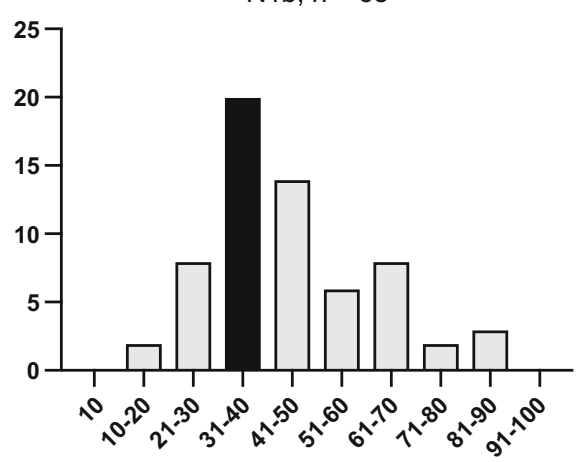

disease form) and smaller tumor size on average, underscoring the importance of lymph node evaluation also in PTMC patients in the clinical work-up. This might as well indicate a different pathophysiological mechanism and tumor biology for the metastases of these tumors, something that warrants further studies which we will initiate on this cohort. Incidence of PTC seems to be increasing more rapidly and the peak age incidence becoming lower than suggested by previous studies. Patients and specifically females with N1b disease were younger than those with N0 or N1a disease. Patients with N1b disease had a lower proportion of females compared to N0 and N1a groups. The reason for the differences between geographic regions regarding the overall incidence of small PTCs and prevalence and distribution of node metastases is also a topic for our further studies.

Acknowledgements Open access funding provided by Umea University. We are grateful to the Scandinavian Quality Register of Thyroid, Parathyroid and Adrenal Surgery (SQRTPA) for collaboration and data supply.

\section{Compliance with ethical standards}

Conflict of interest None of the authors declares any conflicts of interest.
Open Access This article is licensed under a Creative Commons Attribution 4.0 International License, which permits use, sharing, adaptation, distribution and reproduction in any medium or format, as long as you give appropriate credit to the original author(s) and the source, provide a link to the Creative Commons licence, and indicate if changes were made.The images or other third party material in this article are included in the articleã s Creative Commons licence, unless indicated otherwise in a credit line to the material. If material is not included in the articleã s Creative Commons licence and your intended use is not permitted by statutory regulation or exceeds the permitted use, you will need to obtain permission directly from the copyright holder.To view a copy of this licence, visit http://creative commons.org/licenses/by/4.0/.

\section{References}

1. SQRTPA Scandinavian Quality Register for Thyroid, Parathyriod and Adrenal Surgery-Annual Report 2018 (2018). www.sqrtpa. se. Accessed Jan 2019

2. RCC Thyroid Cancer, Swedish National Quality Registry for Thyroid Cancer, Annual Report 2018, (2018). www.cancercen trum.se. Accessed Jan 2019

3. Lundgren CI, Hall P, Ekbom A et al (2003) Incidence and survival of Swedish patients with differentiated thyroid cancer. Int J Cancer 106:569-573

4. Links TP, van Tol KM, Jager PL et al (2005) Life expectancy in differentiated thyroid cancer: a novel approach to survival analysis. Endocr Relat Cancer 12:273-280

5. ATAG Taskforce on Thyroid Nodules, Differentiated Thyroid Cancer, Cooper CS et al (2009) Revised American Thyroid 
Association management guidelines for patients with thyroid nodules and differentiated thyroid cancer. Thyroid 19:1167-1214

6. RCC Thyroid Cancer, Swedish National Guidelines (2018). www.cancercentrum.se. Accessed Jan 2019

7. Pellegriti G, Scollo C, Lumera G et al (2004) Clinical behavior and outcome of papillary thyroid cancers smaller than $1.5 \mathrm{~cm}$ in diameter: a study of 299 cases. J Clin Endocrinol Metab 89:3713-3720

8. Siegel RL, Miller KD, Jemal A (2019) Cancer statistics, 2019. CA Cancer J Clin 69:7-34

9. Roman BR, Morris LG, Davies L (2017) The thyroid cancer epidemic, 2017 perspective. Curr Opin Endocrinol Diabetes Obes 24:332-336

10. Vaccarella S, Franceschi S, Bray F et al (2016) Worldwide thyroid cancer epidemic? The increasing impact of overdiagnosis. N Engl J Med 375:614-617

11. Nikiforov YE (2010) Is ionizing radiation responsible for the increasing incidence of thyroid cancer? Cancer 116:1626-1628

12. Marques P, Leite V, Bugalho MJ (2014) Retrospective analysis of 255 papillary thyroid carcinomas $\leq 2 \mathrm{~cm}$ : clinicohistological features and prognostic factors. Eur Thyroid J 3:258-263

13. Freni F, Galletti B, Galletti F et al (2018) Improved outcomes for papillary thyroid microcarcinoma care: active surveillance and case volume. Ther Adv Endocrinol Metab 9:185-186

14. Lundgren CI, Hall P, Dickman PW et al (2006) Clinically significant prognostic factors for differentiated thyroid carcinoma: a population-based, nested case-control study. Cancer 106:524-531

15. Zhang X, Zhang L, Xue S et al (2019) Predictive factors of lateral lymph node metastasis in solitary papillary thyroid microcarcinoma without gross extrathyroidal extension. Asian $\mathbf{J}$ Surg 42(4):563-570

16. Ito Y, Kudo T, Kobayashi K et al (2012) Prognostic factors for recurrence of papillary thyroid carcinoma in the lymph nodes, lung, and bone: analysis of 5,768 patients with average 10-year follow-up. World J Surg 36:1274-1278. https://doi.org/10.1007/ s00268-012-1423-5

17. Moon JH, Kim JH, Lee EK et al (2018) Corrigendum: author's name correction. Study protocol of multicenter prospective cohort study of active surveillance on papillary thyroid microcarcinoma (MAeSTro). Endocrinol Metab (Seoul) 33:427

18. SCB, Swedish Central Bureau of Statistics (SCB) (2015). www. scb.se. Accessed Jan 2019

19. AJCC (2009) AJCC 7th ed. cancer staging manual

20. ACS Cancer Statistics Center-Thyroid, American Cancer Society (2019).

21. Wang TS, Sosa JA (2018) Thyroid surgery for differentiated thyroid cancer - recent advances and future directions. Nat Rev Endocrinol 14:670-683

22. Gong Y, Yang J, Yan S et al (2018) Pattern of and clinicopathologic risk factors for lateral lymph node metastases in papillary thyroid carcinoma patients with lateral cervical lymphadenopathy. Medicine (Baltimore) 97(36):e12263

Publisher's Note Springer Nature remains neutral with regard to jurisdictional claims in published maps and institutional affiliations. 